\title{
Rentabilidade da monta natural e inseminação artificial em tempo fixo na pecuária de corte
}

Profitability of natural mating and fixed-time artificial insemination in beef cattle

$$
\text { Glauber dos Santos }{ }^{1 *} \text {; Rodrigo Dorneles Tortorella²; Daiane Fausto }{ }^{3}
$$

\footnotetext{
${ }^{1 * 3}$ Programa de Educação Continuada em Economia e Gestão de Empresas [Pecege] - Doutor em Ciências - Rua Alexandre Herculano, 120 - Vila Monteiro - 13418-790 Piracicaba, SP - Brasil<glauber@pecege.com>

${ }^{2}$ Doutor em Ciências Animais - Rua Padre Chagas, 2900 - Centro - 85010-020 - Guarapuava, PR - Brasil
}

\section{Resumo}

O futuro da pecuária de corte e consequentemente sua rentabilidade estão atrelados a otimização do uso da terra, visto que em muitos estados sua área vem sendo ocupada pela agricultura. Uma das alternativas é o uso de biotecnologias da reprodução, como a inseminação artificial em tempo fixo [IATF] que permite a inseminação de animais sem a detecção do estro. O objetivo desse estudo foi avaliar o custo por prenhez da monta natural $[\mathrm{MN}]$ e da IATF em fêmeas pluríparas em um rebanho de corte. Os animais foram divididos em dois grupos: $\mathrm{MN}(\mathrm{n}=150)$ e IATF $(\mathrm{n}=150)$. O custo por prenhez incluiu itens como vida útil do touro, depreciação, remuneração do capital e remuneração de funcionários, entre outros. O custo na MN foi calculado a partir de um valor de compra de $\mathrm{R} \$ 9.000,00$, taxa de prenhez de $75 \%$ e proporção de um touro para 30 vacas. Na IATF foi baseado na taxa de prenhez de $87 \%$ e valor do serviço por animal de R\$ 60,00 . O custo por prenhez foi de R \$117,62 e R\$217,66 para a MN e a IATF, respectivamente. As vantagens da utilização da IATF foram, maior taxa de prenhez no início da estação de monta, antecipação de partos, formação de lotes de bezerros mais pesados a desmama e diminuição do descarte involuntário. Desta forma, apesar de um investimento inicial maior, a IATF teve um bom custo/benefício e rentabilidade.

Palavras-chave: bovinocultura de corte, custo, IATF, MN, prenhez

\begin{abstract}
The future of beef cattle and consequently its profitability are tied to the optimization of land use, since in many states its area has been occupied by agriculture. One of the alternatives is the use of breeding biotechnologies, such as fixed-time artificial insemination [FTAI], which allows the insemination of animals without the detection of estrus. The objective of the present study was to evaluate the pregnancy cost of natural mating [NM] and FTAI in pluriparous females in a beef herd. The animals were divided into two groups: NM ( $\mathrm{n}=150)$ and FTAI $(\mathrm{n}=150)$. The cost per pregnancy included items such as bull's useful life, depreciation, capital remuneration and compensation of employees, among others. The cost in NM was calculated from a purchase value of $\mathrm{R} \$ 9,000.00$, pregnancy rate of $75 \%$ and ratio of one bull to 30 cows. Already at the FTAI, it was based on a pregnancy rate of $87 \%$ and service value per animal of $\mathrm{R} \$ 60.00$. The cost per pregnancy was $\mathrm{R} \$ 117.62$ and $\mathrm{R} \$ 217.66$ for NM and FTAI, respectively. The advantages of using FTAI were higher pregnancy rate at the beginning of the mating season, anticipation of births, formation of heavier calves at weaning and reduction of involuntary discard. In this way, despite a higher initial investment, the FTAI had a good cost / benefit ratio and profitability.
\end{abstract}

Keywords: beef cattle, cost, FTAI, NM, pregnancy

\section{Introdução}

De acordo com os últimos levantamentos, o Brasil possui o maior rebanho bovino comercial do mundo, número este em torno de 210 milhões de cabeças. Ao mesmo tempo a população vem aumentado, assim como a demanda desta por proteína animal, o que acaba por exigir uma maior produção de carne pelos pecuaristas. Em contrapartida, a área total de terra utilizada para a pecuária tem diminuído ano após ano, muito em função do avanço de culturas, como a da soja e milho (IBGE, 2016). Neste contexto, é importante que a pecuária passe por uma modernização e profissionalização a fim de au- mentar a rentabilidade e atratividade da cadeia produtiva de carne, ou seja, na prática, o produtor deve estar preparado para criar um maior número de animais, em menor espaço, com menor custo e maior eficiência.

A reprodução tem papel fundamental na eficiência e rentabilidade do sistema de produção de gado de corte, no qual os baixos índices produtivos estão frequentemente associados à infertilidade prolongada durante o pós-parto, baixo uso de biotecnologias no rebanho e a forma extrativista da pecuária brasileira (Pires et al., 2004). Neste contexto, a monta natural [MN] é o sistema de reprodução mais utilizado na pecuária de corte, con- 
tudo apesar dos avanços no melhoramento genético dos touros, o problema do anestro pós-parto prolongado ainda persiste nas vacas. Esta situação leva a diminuição da eficiência produtiva da fazenda, pois aumenta o intervalo parto-cio, parto-concepção e intervalo entre partos [IEP]. A melhora nos índices reprodutivos e consequentemente produtivos, pode ser alcançada com o uso de biotecnologias da reprodução, como a inseminação artificial em tempo fixo [ATF], que dispensa a detecção do cio e possibilita um maior número de animais inseminados em menor tempo.

A IATF se caracteriza pelo uso de combinações hormonais que objetivam a manipulação e aumento da fertilidade do estro induzido, e da precisão do momento da ovulação (McKinniss et al., 2011; Tortorella et al., 2013). Além das vantagens da Inseminação Artificial [IA] que agrega valores genéticos e econômicos com o uso de touros superiores geneticamente, a IATF concentra a mão-de-obra da propriedade, sincroniza e induz a ciclicidade dos animais. Desta forma, um maior número de vacas é inseminado no início da temporada de monta aumentando a proporção de prenhez, o que possibilita maior tempo de recuperação para nova gestação na temporada seguinte (Sá Filho et al., 2013). Além disso, os bezerros nascidos de IATF no início da temporada de parição são mais pesados ao desmame, aumentando a lucratividade do produtor (Bó et al., 2005).

Independente de qual manejo reprodutivo for adotado na propriedade, sempre haverá custos, seja com hormônios na IATF, com mão-de-obra na inseminação artificial ou com a aquisição de touros na MN. Sendo assim, a decisão por adotar uma ou outra opção de manejo precisa ser tomada a partir de dados técnicos e econômicos, a fim minimizar erros que possam comprometer o desempenho produtivo (Amaral et al., 2003). Neste contexto, é fundamental a análise dos custos diretos e indiretos, sendo estes últimos frequentemente negligenciados pelo produtor, o que leva a uma interpretação equivocada sobre a rentabilidade do sistema de cria. É comum na análise de custos a omissão de informações como custo oportunidade da terra, de capital e depreciação, o que provavelmente provocará a falência a médio e longo prazo de muitas propriedades. Assim, objetivou-se com este trabalho avaliar o benefício:custo do uso da monta natural ou da inseminação em tempo fixo durante a estação de monta de um sistema de produção de pecuária de corte, na região de Guarapuava, PR.

\section{Material e Métodos}

Os dados utilizados para elaboração do trabalho foram baseados em uma fazenda de cria localizada no município de Guarapuava - PR localizado a uma longitude de $25^{\circ} 22^{\prime} \mathrm{S}$ e latitude de $51^{\circ} 52^{\prime} \mathrm{W}$ com 500 ha. O clima predominante na região é o temperado, com inverno e verão bem delimitados e chuvas distribuídas ao longo do ano.

A análise de custos e receitas foi feita baseada nos dados obtidos entre a Estação de Monta [EM] dos meses de dezembro de 2016 a fevereiro de 2017 (90 dias). O plantel era composto de 300 matrizes pluríparas, entre 30 e 60 dias pós-parto, com condição corporal média de 2,5, sendo $1=$ magra e 5 = obesa (Wildman et al. 1982), com bezerro ao pé, das raças Charolesa (Bos taurus) e Canchin (cruza 5/8 Charolês e 3/8 Zebu), representando bem a maioria das fazendas da região quanto ao número de matrizes e tipo racial. Para a análise da rentabilidade as vacas foram divididas em dois grupos, monta natural $(\mathrm{MN}, \mathrm{n}=$ 150) e inseminação artificial em tempo fixo (IATF, $\mathrm{n}=$ 150). Independente do grupo, as vacas foram alocadas em pastagens de Brachiaria decumbens (lotação de 1,2 UA ha $^{-1}$ ) com água e sal mineral ad libitum.

O custo por prenhez na MN utilizou os seguintes parâmetros: número de vacas no sistema de $\mathrm{MN}$ ( $\mathrm{n}=150$ ), vida útil dos touros (seis anos), valor do touro descartado (R\$3.380,00), remuneração do capital ao ano $(\mathrm{R} \$)$, preço do boi gordo em 2017 (R\$140,00@-1), custos operacionais anual por touro $(\mathrm{R} \$ 250,00)$ e aluguel anual da pastagem por touro $(\mathrm{R} \$ 201,60)$. O aluguel da pastagem anual por touro correspondeu a $12 \%$ do valor da arroba (a) do boi gordo multiplicado por 12 meses. Dentro dos custos operacionais foram contabilizados os gastos com alimentação, vacinação, exame andrológico, vermifugação e mão de obra. O valor do touro descarte foi baseado no peso final de abate em arrobas (26@) multiplicado pelo valor da@da vaca gorda (R\$130,00). Para o cálculo da remuneração capital ao ano, em reais, foi utilizada a taxa Selic média de 12\%. Dentro da propriedade o número de touros utilizados também influenciou no custo por prenhez, sendo que na fazenda em questão, a proporção de um touro para 30 vacas foi respeitada. Desta forma, foram utilizados cinco touros da raça Angus com valor unitário de compra de $\mathrm{R} \$ 9.000,00$. Além disto, foi estimada uma taxa de prenhez de $20 \%, 30 \%$ e $75 \%$ aos 30 dias, 60 dias e final da EM, respectivamente (Sá Filho et al. 2013). O custo por prenhez/touro foi obtido através da seguinte eq. (1):

$$
\mathrm{CP}=\frac{\left(\mathrm{CTo} \times \mathrm{n}^{\circ} \mathrm{T}\right)}{\mathrm{TFP}}
$$

onde, CP: custo por prenhez; CTo: custo por touro; $\mathrm{n}^{\circ} \mathrm{T}$ : número de touros; e TFP: total de fêmeas prenhes

O custo por touro foi calculado pela eq. (2):

$$
\mathrm{CTo}=\mathrm{Dp}+\mathrm{AP}+\mathrm{CO}+[(\mathrm{Dp}+\mathrm{AP}+\mathrm{CO}+\mathrm{VC}) \times \mathrm{RC}]
$$

onde, CTo: custo por touro; DP: depreciação; AP: aluguel de pastagem; CO: custo operacional; VC: valor da compra do touro; e RC: remuneração do capital 
A análise do custo por prenhez com a IATF $(n=150)$ foi baseada no modelo seguido pela maioria das fazendas que contratam este serviço na região, no qual o veterinário cobra um valor fechado por vaca inseminada. $\mathrm{O}$ valor, em torno de $\mathrm{R} \$ 60,00$, incluiu mão de obra (aplicação de hormônios e inseminação, R \$ 20,00), sêmen $(\mathrm{R} \$ 20,00)$, hormônios e material descartável $(\mathrm{R} \$ 20,00)$. Neste caso, a fazenda não teve custos com depreciação de materiais como o botijão de sêmen, ou operacionais como a manutenção do nível do nitrogênio líquido dentro do botijão.

Outro item de custo foi a remuneração do capital ao ano, em reais, em que foi utilizada a taxa Selic média de $12 \%$. Foi utilizado um protocolo hormonal em que os animais passaram três vezes pelo curral, incluindo o dia da inseminação. O tempo de serviço total dos manejos (dez horas) serviu de base para a análise do custo da mão de obra da fazenda, em função do salário funcionário. Foi utilizado um salário base de $\mathrm{R} \$ 6,25$ por hora adicionados os encargos. Contudo, como na segunda e terceira inseminação o tempo de serviço foi menor, utilizou-se uma média aritmética.

No grupo IATF foram realizadas até três inseminações por vaca, ou seja, no exame ultrassonográfico 30 dias após IATF, as vacas vazias eram ressincronizadas e inseminadas novamente. Desta forma, o touro não foi utilizado, totalizando uma EM de 80 dias. Sales et al. (2013) relata uma taxa de prenhez média por IATF de $50 \%$ o que resultaria em uma taxa de prenhez de $87 \%$ ao final da EM. O custo por prenhez/IATF foi calculado pela eq. (3):

$$
\mathrm{CP}=\frac{\left(\mathrm{CTr} \times \mathrm{n}^{\circ} \mathrm{IATF}\right)}{\mathrm{TFP}}
$$

onde, CP: custo por prenhez; CTr, custo por tratamento; $n^{\circ}$ IATF: número de inseminações em tempo fixo; e TFP: total de fêmeas prenhes.

O custo por tratamento foi calculado pela eq. (4):

$$
\mathrm{CTr}=\mathrm{VF}+\mathrm{RF}+[(\mathrm{VF}+\mathrm{RF}) \times \mathrm{RC}]
$$

onde, CTr: custo por tratamento; VF: valor fixo; RF: remuneração dos funcionários; e RC: remuneração do capital.

Independente do grupo experimental, não foram contabilizadas as despesas com depreciação de vacas, das vacinas, dos medicamentos, juros do capital imobilizado (matrizes, terra, máquinas e instalações) e juros do capital de custeio. Para Arruda (1993), enquanto a vaca estiver com o bezerro ao pé estes custos estão sendo creditados aos bezerros, ou seja, a contabilização destes custos só é válida após o desmame.

\section{Resultados e Discussão}

A partir da análise dos resultados foi possível observar que o custo por prenhez foi de $\mathrm{R} \$ 117,62$ e $\mathrm{R} \$ 217,66$ para os grupos MN e IATF, respectivamente. Contudo, esta diferença pode ser maior ou menor a depender de alguns fatores como proporção de touro por vacas e custo de aquisição do touro. Na tabela 1 realizou uma simulação do custo por prenhez de acordo com preço do touro e proporção de touro para vacas, partindo de uma taxa de prenhez final de $75 \%$. Foi possível observar que a partir de uma taxa fixa de prenhez quanto maior o custo de aquisição de um touro seria interessante otimizar seu uso em um maior número de fêmeas para diluir o custo por prenhez. Para tanto, é importante que seja feita um exame andrológico prévio a EM para atestar a saúde geral e reprodutiva do macho.

Tabela 1. Custo por prenhez, em reais, da monta natural de acordo com o preço do touro e da relação touro:vacas, em uma taxa de prenhez final da estação de monta de $75 \%$

\begin{tabular}{rcrc}
\hline Preço do & \multicolumn{3}{c}{ Relação touro:vaca } \\
\cline { 2 - 4 } touro & $1: 25$ & $1: 30$ & $1: 50$ \\
\hline--- R $\$---$ & & & \\
$7.000,00$ & 108,29 & 90,24 & 54,14 \\
$9.000,00$ & 141,15 & 117,62 & 70,57 \\
$11.000,00$ & 174,01 & 145,00 & 87,00 \\
\hline
\end{tabular}

A diferença de $\mathrm{R} \$ 100,00$ a mais para o custo da prenhez quando usada a IATF pode deixar de existir quando por alguma situação o touro vem a óbito (taxa de mortalidade de 1-3\%) ou perde a capacidade de serviço durante a EM. Além disto, em algumas situações o uso do touro pode ser um complicador para o manejo da propriedade, pois o mesmo necessita de uma pastagem específica para ele quando fora da EM. Além disto, devido ao temperamento mais agressivo em função da elevada concentração de testosterona, pode ocasionar quebra de cerca ou até mesmo injúrias nos funcionários.

Na tabela 2, mantendo-se a proporção de um touro para 30 vacas, foi colocado outro fator que interfere no custo por prenhez, a taxa de gestação ao final da EM. Foi possível visualizar que quanto menor a taxa de prenhez ao final da EM maior será o custo para produzir um bezerro. O Brasil tem uma taxa de natalidade em torno de $60 \%$ e um intervalo entre partos de 21 meses, enquanto que em sistemas mais tecnificados os mesmos índices estão em torno de $80 \%$ e 12 a 13 meses, respectivamente (Kichel et al., 2011). Uma das razões para a baixa taxa de natalidade, que aumentou o custo por prenhez, foi a baixa eficiência reprodutiva das vacas com cria ao pé (Yavas e Walton, 2000). Isto deve-se ao fato de que quando não estimuladas artificialmente acabam demonstrando o estro somente no final da EM ou até mesmo após sua 
finalização, ou seja, o macho pode estar apto a reprodução, porém a fêmea não demonstra interesse em aceitar a monta. Este fator fisiológico da fêmea com cria ao pé, é agravado pelas condições das pastagens brasileiras, as quais estima-se que em torno de $80 \%$ estão em vias de degradação.

Tabela 2. Custo por prenhez, em reais, da monta natural de acordo com o preço do touro e da taxa de prenhez final da estação de monta mantendo relação de um touro para 30 vacas

\begin{tabular}{|c|c|c|c|}
\hline \multirow{2}{*}{$\begin{array}{l}\text { Preço do } \\
\text { touro }\end{array}$} & \multicolumn{3}{|c|}{ Taxa de Prenhez } \\
\hline & 60 & 75 & 90 \\
\hline ---- R\$ ---- & ------------- & $\%$ & -1----- \\
\hline $7.000,00$ & 112,30 & 90,24 & 74,87 \\
\hline $9.000,00$ & 146,38 & 117,62 & 97,58 \\
\hline $11.000,00$ & 180,45 & 145,00 & 120,30 \\
\hline
\end{tabular}

Apesar do maior custo por prenhez no grupo IATF observou-se uma maior taxa de prenhez em relação a MN (130 e 112). A partir deste resultado, foi possível fazer uma previsão de receita no momento da venda do bezerro desmamado com 6 a 8 meses e $240 \mathrm{~kg}$ a um preço de $\mathrm{R} \$ 7,00 \mathrm{o} \mathrm{kg}$ do bezerro desmamado. A receita usando a IATF seria em torno de $\mathrm{R} \$ 13.440,00$ maior do que se fosse usada a MN. Além disto, é comum o descarte de fêmeas vazias ao final da estação de monta, descarte involuntário, o que gerou um custo indireto de reposição do plantel com aquisição de novilhas ou diminuição das vendas de bezerras desmamadas, ou seja, uma parcela das bezerras que poderiam estar sendo vendidas e gerando receita, deverá permanecer no rebanho para repor este número extra de fêmeas vazias a serem descartadas. Fazendo uma simulação, no grupo MN seriam descartadas 18 vacas a mais que no grupo IATF, a um valor da (a) da vaca gorda em R $\$ 120,00$ e um peso de abate de $16 @$ seria gerado de receita $\mathrm{R} \$ 34.560,00$. Contudo, para a aquisição de 18 novilhas seria desembolsado por animal um valor de $\mathrm{R} \$ 1.950,00$ partindo do preço base na região de $\mathrm{R} \$ 6,00$ por $\mathrm{kg}$ de peso vivo para uma novilha com média de $325 \mathrm{~kg}$, ou seja, para as 18 novilhas seriam necessários R \$37.050,00 sendo um valor maior do que o recebido pela venda das vacas de descarte.

Amaral et al. (2003) realizaram simulações para comparar o custo de prenhez do touro versus a IA convencional versus a IATF. Os mesmos concluíram que o touro apresentava o menor custo seguido da IA convencional e da IATF. Segundo estes, a IATF teve no custo dos hormônios e relativa baixa taxa de prenhez $(\sim 50 \%)$ os principais fatores que aumentaram o investimento para sua realização.

Na tabela 3 pode ser observado que quando a taxa de prenhez da IATF foi maior ou o custo do sêmen foi menor, o custo por prenhez acaba diminuindo. Para exemplificar, quando a taxa de prenhez ao final da EM sobe 5\% (87\% para 93\%) usando um sêmen de $\mathrm{R} \$ 20,00$, o custo por prenhez diminuiu em torno de $20 \%$ ( $\mathrm{R} \$$ 217,66 versus R\$180,51). Sá Filho et al. (2013) encontraram uma taxa de prenhez de $7 \%$ a $10 \%$ maior no final da EM quando comparam lotes que usaram IATF e outros que usaram IA convencional ou MN.

Tabela 3. Custo por prenhez, em reais, da inseminação artificial em tempo fixo [IATF] de acordo com o preço do sêmen e da taxa de prenhez final da estação de monta após três IATFs

\begin{tabular}{|c|c|c|c|}
\hline \multirow{2}{*}{$\begin{array}{l}\text { Preço do } \\
\text { sêmen }\end{array}$} & \multicolumn{3}{|c|}{$\begin{array}{c}\text { Taxa de Prenhez do lote após três } \\
\text { IATF }\end{array}$} \\
\hline & 78 & 87 & 93 \\
\hline $\mathrm{R} \$$ & - & $\%$ & -------- \\
\hline 15,00 & 258,82 & 206,00 & 172,15 \\
\hline 20,00 & 271,38 & 217,66 & 180,51 \\
\hline 25,00 & 283,94 & 226,00 & 188,87 \\
\hline
\end{tabular}

Silva et al. (2007) avaliaram o custo benefício da IA convencional versus a IATF em vacas mestiças com 60 dias após o parto e verificaram que o custo por prenhez foi menor na IA convencional. Contudo, nem todas as vacas do grupo IA convencional puderam ser inseminadas visto que não demonstraram estro. Além disto, foram necessários 35 dias para inseminar todo lote da IA, enquanto que na IATF todas foram inseminadas em um dia. Desta forma, a antecipação da prenhez gerou uma economia financeira, pois o custo da vaca vazia foi maior do que o da vaca prenhe uma vez que o futuro bezerro irá gerar uma receita extra.

Uma das grandes vantagens da IATF em relação a MN foi a antecipação da prenhez, o que levou a um maior tempo de recuperação da fêmea após o parto para a próxima EM, aumentou a probabilidade de nova prenhez e diminuiu a taxa de descarte (Sá Filho et al., 2013). A causa para a maior taxa de prenhez no início da EM foi a indução da ovulação em vacas em anestro pós-parto que demoraram um certo tempo para retornar a ciclicidade. $\mathrm{O}$ anestro pós-parto, período sem demonstração do estro, é uma fase fisiológico de recuperação endócrina da fêmea, entretanto quando prolongado acaba por diminuir a eficiência reprodutiva e produtiva da pecuária (Yavas e Walton, 2000). Desta forma, no início da EM muitas fêmeas não demonstraram estro, e inclusive, algumas não foram até o final, o que diminuiu as possibilidades da MN.

A antecipação da prenhez com formação de lotes de bezerros também se mostrou interessante no momento da desmama. Aqueles advindos da IATF além de terem maior padrão morfológico, apresentaram maior peso o que aumentaria a rentabilidade do produtor que trabalha com a cria. Bó et al. (2005) observaram que os bezerros da IATF eram em torno de 10 a $20 \mathrm{~kg}$ mais pesados do 
que os da MN. Duas causas podem ser relacionadas a este fato, sendo a primeira a antecipação dos partos o que aumentaria o tempo para o crescimento do bezerro e a segunda relacionada a maior qualidade genética do sêmen do touro da IATF. Em uma simulação com o valor do peso, em kg, do bezerro desmamado em $\mathrm{R} \$ 7,00$ seria recebido de $R \$ 70,00$ a $R \$ 140,00$ a mais no momento da venda de cada bezerro. Segundo Neto e Dalchiavon (2017), o uso de inseminação artificial tempo fixo na pecuária de corte é um projeto que tem viabilidade econômica, desde que sejam considerados os valores com a venda de matrizes ao término do projeto.

A partir do investimento inicial de $\mathrm{R} \$ 100,00$ maior no grupo IATF por prenhez, quando este foi subtraído das receitas extras com o maior peso a desmama $(\mathrm{R} \$ 105,00)$ e taxa de prenhez ao final da estação de monta $(\mathrm{R} \$ 1680,00 * 10 \%=\mathrm{R} \$ 168,00)$ encontra-se um valor de - R\$173,00, ou seja, o resultado econômico pode ser satisfatório mesmo com um investimento inicial maior. Contudo, mesmo apresentadas algumas vantagens da IATF, não é recomendado que seja executada sem um planejamento nutricional para as fêmeas e um bom sistema de gestão zootécnica e financeira na propriedade. Além disto, na primeira execução desta biotecnologia recomenda-se a formação de lotes pequenos, em torno de 50 fêmeas, o que irá permitir um amadurecimento na execução das atividades por parte da equipe de trabalho da fazenda. Esta biotecnologia também é viável para pequenos criadores (10 a 20 fêmeas), principalmente neste método de contratação do serviço completo, o que eliminaria as despesas com depreciação de material.

\section{Conclusão}

A estratégia reprodutiva com monta natural apresenta um custo inicial menor do que a inseminação em tempo fixo. No entanto, quando levado em consideração outras vantagens zootécnicas, a IATF obtém melhor custo/benefício, principalmente em fêmeas que não estão ciclando regularmente. Ressalta-se que outros cuidados como correta alimentação e manejo sanitário dos animais são fundamentais para que a técnica de IATF possa apresentar os resultados econômicos positivo.

\section{Referências}

Amaral, T.B.; Costa, F.P.; Corrêa, E.S. 2003. Touros melhoradores ou inseminação artificial: um exercício de avaliação econômica. Embrapa/CNPGC, Campo Grande, MS. $\mathrm{n}^{\circ} 140$.

Arruda, Z.J. 1993. Considerações econômicas sobre a produção de bezerros de corte. Embrapa-CNPGC, Campo Grande, MS. n ${ }^{\circ} 47$.

Bó, G.A.; Cutaia, L.; Chesta, P.; Balla, E.; Picinato, D.; Peres, L.; Maranã, D.; Avillés, M.; Menchaca, A.; Veneranda, G.; Baruselli, P.S. 2005. Implementacion de programas de inseminación artificial en rodeos de cria de argentina. Proc VI Simposio
Internacional de Reproducción Animal. Córdoba, Argentina, p. $97-128$.

Instituto Brasileiro de Geografia e Estatística [IBGE]. 2016. Pesquisa Pecuária Municipal. Disponível em: <www.sidra. ibge.gov.br>. Acesso em: 12 jul. 2017.

Kichel, A.N.; Costa, A.A.A.; Verzignassi, J.R.; Queiroz, H.P. 2011. Diagnóstico para o planejamento da propriedade. Embrapa/CNPGC, Campo Grande, MS. nº182.

McKinniss, E.N.; Esterman, R.D.; Woodall, S.A.; Austin, B.R.; Hersom, M.J.; Yelich, J.V. 2011. Evaluation of two progestogen-based estrous synchronization protocols in yearling heifers of Bos indicus $\times$ Bos taurus breeding. Theriogenology 75:1699-1707.

Neto, N.Z e Dalchiavon, F.C. Viabilidade financeira da inseminação artificial em tempo fixo de bezerros cruzados Nelore e Aberdeen Angus. Revista IPecege 3(3): 23-27, 2017

Pires, V.A.; Araujo, C.R.; Mendes, Q.C. 2004. Fatores que interferem na eficiência reprodutiva de bovinos de corte. In: SIMPÓSIO PECUÁRIA INTENSIVA NOS TRÓPICOS. Anais... Piracicaba: Fundação de Estudos Agrários Luiz de Queiroz, p. 355-398.

Sá filho, M.F.; Penteado, L.; Reis, E.L.; Reis T.A.; Galvão, K.N.; Baruselli, P.S. 2013. Timed artificial insemination early in the breeding season improves the reproductive performance of suckled beef cows. Theriogenology 79:625-632.

Silva, A.S.; Silva, E.V.C.; Nogueira, E.; Zúccari, C.E.S.N. 2007. Avaliação do custo/benefício da inseminação artificial convencional e em tempo fixo de fêmeas bovinas pluríparas de corte. Revista Brasileira de Reprodução Animal 31: 443:455.

Tortorella, R.D.; Ferreira, R.; Dos Santos, J.T.; Neto, O.S.A.; Barreta, M.H.; Oliveira, J.F.; Gonçalves, P.B.; Neves J.P. 2013. The effect of equine chorionic gonadotropin on follicular size, luteal volume, circulating progesterone concentrations, and pregnancy rates in anestrous beef cows treated with a novel fixed-time artificial insemination protocol. Theriogenology 79: 1204-1209.

Yavas, Y.; Walton, J.S. 2000. Postpartum acyclicity in suckled beef cows: A review. Theriogenology 54: 25-55.

Wildman, E.E.; Jones, G.M.; Wagner, P.E. 1982. A dairy cow body condition scoring system and its relationship to selected production characteristics. Journal of Dairy Science 65: 495-501. 